\title{
Lernen in Lebensphasen - eine Alternative zum Lebenslangen Lernen?
}

\author{
Burkhard Schäffer • Olaf Dörner · Franz Krämer
}

Eingegangen: 11. Mai 2015 / Angenommen: 11. Juli 2015 / Online publiziert: 3. September 2015

(C) Die Autor(en) 2015. Dieser Artikel ist auf Springerlink.com mit Open Access verfügbar.

Zusammenfassung Die Beteiligung Erwachsener am Lebenslangen Lernen entspricht bei Weitem nicht den Erwartungen, die mit dem Programm verbunden sind. Gründe dafür sind vielfältig und unterschiedlich. Unsere These ist, dass sich Erwachsene in ihren Lernaktivitäten an Lebensphasen orientieren. Die Beteiligung an institutionalisierten Lernarrangements von Weiterbildung ist demnach weniger eine Sache der Akzeptanz Lebenslangen Lernens, sondern vielmehr eine, die in Bezug auf soziokulturelle Erwartungsmuster lebensphasenabhängig entschieden wird. Vor allem in der Erwerbsphase werden Weiterbildungsorientierungen konstituiert durch berufliche und betriebliche Positionen und deren habituelle Wahrnehmung. Wie werden Chancen und Möglichkeiten von Weiterbildung wahrgenommen und gedacht? Weiterbildung erweist sich dann als etwas, für das sich selbstverständlich entschieden werden kann, als etwas Unerreichbares, Last, Zumutung oder auch als unerheblich. Im Beitrag untermauern wir die These von der Lebensphasenabhängigkeit theoretisch unter Bezugnahme auf die Differenzierung von Biografie, Lebenslauf und Lebensverlauf sowie mit empirischen Befunden zu Lern- und Weiterbildungsorientierungen der Babyboomer.

Prof. Dr. B. Schäffer $(\bowtie) \cdot$ Dipl.-Päd. F. Krämer

Universität der Bundeswehr München,

Werner-Heisenberg-Weg 39,

85579 Neubiberg, Deutschland

E-Mail: Burkhard.schaeffer@unibw.de

Dipl.-Päd. F. Krämer

E-Mail: f.kramer@unibw.de

Prof. Dr. O. Dörner

Otto-von-Guericke-Universität Magdeburg,

Zschokkestraße 32,

39104 Magdeburg, Deutschland

E-Mail: olaf.doerner@ovgu.de 
Schlüsselwörter Lernen Erwachsener · Weiterbildung · Lebensphase ·

Babyboomer - Altersbilder - Weiterbildungsorientierungen - Lebenslanges Lernen

\section{Life-stage-dependent Learning: an Alternative to Lifelong Learning?}

Abstract For various reasons adult participation in lifelong learning does not measure up to expectations tied to the agenda of lifelong learning. The article suggests that adults relate learning activities to their conceptions of life stages. Therefore, participation in institutionalized continuing education must rather be linked to lifestage-dependant decisions based on sociocultural patterns than to the acceptance of lifelong learning in general. Specifically in the stage of professional life, these orientations towards continuing education are formed by habitual perceptions of one's own occupational and operational position. How do adults experience chances and possibilities of continuing education then? Continuing education is seen as a matter of course, as unattainable, as unreasonable or as irrelevant. The article underpins the supposition of life-stage-dependancy theoretically by differentiating between biography, life course as triadic sociocultural pattern and life course as category for longitudinal cohort research. Our assumption is also substantiated by empirical findings about baby boomers' orientations towards learning and continuing education.

Keywords adult learning $\cdot$ continuing education $\cdot$ life stage $\cdot$ baby boomer images of aging $\cdot$ orientations towards continuing education $\cdot$ lifelong learning

\section{Einleitung}

Lernen Erwachsene ihr ganzes Leben lang kontinuierlich oder hat Lernen in bestimmten Phasen des Lebens Konjunktur? Für das Programm Lebenslanges Lernen und dessen Verwirklichung ist dies eine entscheidende Frage. Zunächst ist Lernen im Sinne von „Erfahrungen machen“ nicht zu umgehen und insofern eine Selbstverständlichkeit: Jede/r Erwachsene macht sein/ihr ganzes Leben lang biografische Erfahrungen, die sich als biografische Lern- und Bildungsprozesse niederschlagen. Dies kann dann empirisch differenziert und theoretisch analysiert werden (vgl. hierzu jüngst: Nohl et al. 2015). In dem vorliegenden Beitrag wird die Frage spezifischer, nämlich im Hinblick auf institutionalisierte, ,formale“ und „,non-formale“ Kontexte des Lernens im Erwachsenenalter (im Sinne der UNESCO Definition) akzentuiert. Zwar bilden nicht-intentionale (biografische) Lern- und Bildungsprozesse eine wichtige Voraussetzung für institutionelles Lernen, sind aber nicht primär Gegenstand dieses Beitrages. Vielmehr geht es uns um die Frage danach, inwieweit sich die Inanspruchnahme institutionalisierter Lernsituationen im Kontext von Erwachsenenbildung/Weiterbildung (EB/WB) in Abhängigkeit von Lebensphasen gestaltet. Wir gehen davon aus, dass Erwachsene Entscheidungen zur Teilnahme an EB/WB aufgrund von Weiterbildungsorientierungen treffen, die wiederum eingebettet sind in Vorstellungen über die Bedeutung von Lernen in unterschiedlichen Lebens- und Altersphasen. Welche Rolle spielt also das Verhältnis von der Wahrnehmung der eigenen Lebensphase einerseits 
und Weiterbildungsorientierungen andererseits für die Entscheidung, an Weiterbildung teilzunehmen oder auch nicht? Fühlt man sich bspw. als 50-Jähriger zu alt zum Lernen in institutionalisierten Kontexten oder traut man es sich noch zu? Schließlich ist auch die Einbettung dieser Weiterbildungsorientierungen in allgemeinere, milieu-, generations- und/oder geschlechtsspezifische Lern- und Bildungsorientierungen im Erwachsenenalter zu berücksichtigen.

In diesem Beitrag vertreten wir die These, dass sich Lernen Erwachsener im Rahmen von formaler und non-formaler Weiterbildung nach wie vor in dem Sinne lebensphasenabhängig vollzieht, als sich Subjekte in ihren biografischen Entscheidungen an der soziokulturellen Konstruktion eines „Normallebenslaufs" mit seiner Einteilung in Ausbildungs-, Erwerbs- und Nacherwerbsphase orientieren. Programmatische Konzepte Lebenslangen Lernens dagegen gehen implizit von einer De-Institutionalisierung des Lebenslaufs und seiner orientierenden Kraft im Hinblick auf Lern- und Bildungskontexte aus, stehen hierzu also im Widerspruch.

Um diese These zu belegen, werden wir zunächst (1) Lebensphasenabhängigkeit im Kontext unterschiedlicher Konzepte diskutieren und auf Weiterbildungskontexte beziehen sowie auf unsere Überlegungen auf die Lebensphase zwischen 50 und 60 Jahren fokussieren. Auf dieser „Plateauphase“ befinden sich momentan die Kohorten der Babyboomer (*1954-1964). Nach der Skizzierung methodischer Aspekte (2) werden wir eine empirisch basierte und lebensphasenorientierte Typologie zur Weiterbildungsbeteiligung der Babyboomer vorstellen (3). Abschließend stellen wir „Lernen in Lebensphasen“" als empirisch fundierte Alternative dem ,lebenslangen Lernen“ gegenüber (4).

\section{Lebensphasen, Lebenslanges Lernen durch Weiterbildung und Altersbilder der Babyboomer}

Mit dem Terminus der Lebensphase wird auf eine Debatte Bezug genommen, die eine ihrer Ausprägungen in der Dichotomie Lebenslauf versus Biografie gefunden hat (vgl. jüngst: Maier-Gutheil 2015). Demnach steht Biografie für die subjektbezogene, sinnstiftende Verarbeitung durch ein Individuum und Lebenslauf für die ,objektive Datenspur", die dieses Individuum durch sein Durchschreiten unterschiedlicher Altersphasen (Kindheit, Jugend, Erwachsenheit, Alter) und Dimensionen (Familie, Schule, Ausbildung, Beruf, Freizeit etc.) innerhalb eines Lebenslaufs erzeugt. Nach unserem Dafürhalten wird mit dieser Zweiteilung die Differenzierung zwischen Lebenslauf und Lebensverlauf verdeckt, die beide zur Biografie in einem Spannungsverhältnis stehen. Lebenslauf ist, wie Martin Kohli (1985) gezeigt hat, auf Prozesse der Institutionalisierung und De-Institutionalisierung von kollektiven soziokulturellen Erwartungshorizonten im Hinblick auf eine normalbiografische Ausformung bezogen. Im Zuge der „Institutionalisierung des Lebenslaufs“ (ebd.) hat sich in westlichen Industriegesellschaften nach dem II. Weltkrieg ein soziokulturelles biografisches Erwartungsschema herausgebildet, das auf eine Dreiteilung des Lebenslaufs in Vorerwerbs-, Erwerbs- und Nacherwerbsphase hinausläuft. Ein Lebensverlauf dagegen bezieht sich nicht auf solche soziokulturellen Erwartungshorizonte, sondern ist auf die Analyse von typischen Ereigniszeitpunkten (Heirat, erstes Kind, Erwerbseintritt 
etc.) und die Dauer von Ereignissen (Studium, Elternschaft, Verbleib in Erwerbsphase u. Ä.) im Verlauf des Lebens großer Geburtskohorten bezogen (vgl. Mayer und Diewald 2007). Die Differenzierung von Lebenslauf als Institution und soziokulturellem Erwartungsschema, Lebensverlauf als seinem quantitativen Pendant bei der Analyse großer Kohorten und Biografie als individueller qualitativer Verarbeitungs- und Sinnbildungsprozess bildet insofern u. E. einen angemessenen Rahmen für die Analyse von phasenbezogenen Lern- und Bildungsprozessen im Erwachsenenalter.

\subsection{Das Konzept biografischer Lebens- und Lernphasen}

Lebensphasenmodelle aus Psychologie, Soziologie und Pädagogik spielen eine wichtige Rolle bei der Beschäftigung mit der Frage nach der Bedeutung von Lern- und Bildungsprozessen in der Humanontogenese. In solchen Modellen werden Lern- und Ausbildungszeiten überwiegend im Kindes- und Jugendalter verortet; institutionalisierte Lernprozesse Erwachsener werden dagegen eher selten thematisiert. Exemplarisch seien hier Eriksons 3-stufiges Entwicklungsmodell der Identität des Erwachsenen (ders. 1991), Havighursts differenzierteres Konzept der „Entwicklungsaufgaben“ (Havighurst 1972) oder anthropologisch orientierte Phasenmodelle, wie das von Franz Pöggeler über die „Entwicklungs- und Bildungsphasen des Erwachsenen“ (Pöggeler 1964, S. 73 f.) angeführt. Bei aller Ausdifferenzierung lässt sich der Grundgedanke von biografischen Phasenmodellen wie folgt umschreiben: Bestimmte biografische Entscheidungen und daraus resultierende Handlungen werden, ,üblicherweise“ in einer bestimmten Altersphase getroffen und vollzogen - z. B. heiraten, Kinder bekommen, eine Ausbildung absolvieren, arbeiten oder aus dem Berufsleben ausscheiden etc. Hieraus werden dann auch temporale Perspektiven auf die eigene Biografie und die von anderen abgeleitet: Biografische Ereignisse werden als ,zu früh“, ,gerade recht“ oder „,zu spät“ bei einem selbst erfahren bzw. bei anderen gerahmt. Es wird zwar nicht in Abrede gestellt, dass in der Erwerbs- und Nacherwerbsphase auch gelernt werden kann; als soziokulturelle Erwartung im Sinne einer „Normalbiografie“(s. o.) wird dies jedoch nicht an den/die Einzelne/n herangetragen.

Phasenmodelle insgesamt wurden kritisiert für ihre implizite Normativität, die in ihrer Orientierung an der „Normalität“ männlicher Mittelstandsbiografien in westeuropäischen und nordamerikanischen Gesellschaften zum Ausdruck kam. Auch aus Sicht der Psychologie der Lebensspanne werden Phasenmodelle u. a. mit dem Argument abgelehnt, dass Unterschiede zwischen vermeintlich gleichen Altersphasen größer sind, als zwischen Personen unterschiedlichen Alters (Brandtstädter 1990). Stattdessen wird ein Rahmenmodell der ,generellen Architektur des Lebensverlaufs“ formuliert, das zwar von bestimmten invarianten biologischen und kulturellen Vorgaben ausgeht, die aber nicht in Phasen, sondern über die gesamte Lebensspanne hinweg in unterschiedlicher Intensität wirksam werden. Die Vorgaben lassen sich in drei Thesen zusammenfassen: Evolutionäre Selektionsvorteile nehmen mit dem Alter ab, der Bedarf an Kultur nimmt mit dem Alter zu, während ihr Wirkungsgrad mit dem Alter abnimmt (Lindenberger 2002, S. 350 f.; siehe auch Brandtstädter 2001; Überblick bei Leipold 2012). Aus soziologischer Perspektive standen Phasenmodelle vor dem Hintergrund der Individualisierungstheorie (Beck 1986) wegen ihrer Rigidität und Inflexibilität in der Kritik. Zunehmend wurde eine „Deinstitutionalisierung“ des 
Lebenslaufs, also die Erosion von herkömmlichen Lebensablaufmustern hin zu einem ,anything goes“" konstatiert (exemplarisch: Beck und Beck-Gernsheim 1993).

\subsection{Weiterbildung lebenslang oder in bestimmten Lebensphasen?}

Es ist u. E. kein Zufall, dass sich parallel zum Aufkommen der lebensspannentheoretischen Ansätze und der Individualisierungsthese in den 1990er Jahren programmatische Konzepte des Lebenslangen Lernens durchsetzten, die die Erosion der Dreiteilung des Lebenslaufs auch im Bereich von formellem, non-formalem und informellem Lernen im Erwachsenenalter behaupteten (exemplarisch: Dohmen 1996). Die Idee des Lebenslangen Lernens selbst ist schon älter (vgl. Kade und Seitter 1996; Kraus 2001; Hof 2009; Kuhlenkamp 2010; Rausch 2015). Sie läuft auf ein permanentes, in Teilen lebensphasenunabhängiges Neu- und Umlernen über das gesamte Erwachsenenalter hinaus, das durch Weiterbildungsorganisationen unterstützt werden müsse, die wiederum selbst der Förderung bedürfen (Kuhlenkamp 2010, S. 122 ff.). Entgegen der Programmatik ist nun zu beobachten, dass sich Lebenslanges Lernen nicht flächendeckend durchgesetzt hat. Aktuell liegt die Beteiligungsquote an Weiterbildung bei etwa 50\% (vgl. Bilger und Kuper 2013, S. 30) Zudem sind es vor allem 35- bis 49-Jährige, die an Weiterbildung teilnehmen (vgl. Leven et al. 2013, S. 82 f.). Insofern spricht einiges dafür, den Blick auf Lebensphasen zu richten. Bei Studien, denen eine lebensphasenbezogene Idee zugrunde liegt, etwa Lernen in der Nacherwerbsphase (vgl. Friebe et al. 2014), wird Lernen vielfältig ausbuchstabiert. Es werden z. B. Eingruppierungen nach chronologischem Alter oder auch der Dualismus „Beruflichkeit versus Nicht-Beruflichkeit“ als Ordnungskategorie eingesetzt. Der Letztgenannte verweist auf klassisch-triadische Lebenslaufregimes (Vorerwerbs-, Erwerbs- und Nacherwerbsphase), die auch in die lebensphasenbezogene Organisation biografischen Lernens hineinwirken.'

Weiterbildung als lebensphasenabhängiges Phänomen lässt sich auch anhand von Befunden repräsentativer Studien zu Weiterbildung und Lebenslangem Lernen belegen. Sie zeigen, dass Weiterbildung in betrieblichen Zusammenhängen gegenüber anderen Zusammenhängen deutlich dominiert (vgl. Bilger und Kuper 2013, S. 45) und lediglich in der Erwerbsarbeitsphase Kontinuitäten aufweist (vgl. Schiersmann 2006). Die wichtigsten Lernkontexte (im Rahmen des Lebenslangen Lernens) drücken berufliche Gelegenheitsstrukturen der Erwerbsarbeit aus, nämlich solche, die im Zusammenhang mit Berufsbiografie und beruflichen Status stehen (vgl. Baethge und Baethge-Kinsky 2004, S. 70 f.). Mehr noch: Weiterbildung wird darüber hinaus von den Befragten mehrheitlich als beruflich akzeptierter Zwang oder inakzeptable Zumutung verstanden (vgl. ebd., S. 59). Mit Blick auf den Faktor Alter ist festzuhalten, dass Beteiligung von über 50-Jährigen an Weiterbildung in formaler, non-formaler und auch informeller Form weniger ausgeprägt ist als bei jüngeren Altersgruppen (vgl. etwa Leven et al. 2013, S. 83; Kuwan und Seidel 2013, S. 270). Einer Studie von Schiersmann (2006, S. 45) folgend geben lediglich 31\% derjenigen zwischen 50 und 64 Jahren an, überhaupt einen Bedarf an Weiterbildung zu haben. Bei den Jüngeren sind höhere Zustimmungswerte zu verzeichnen (19 bis 34 Jahre: $61 \%$ und

${ }^{1}$ Vgl. mit Bezug auf weibliche Führungskräfte im Bildungsbereich: Schlüter 2010, S. 5 f. 
35 bis 49 Jahre: 54\%, ebd.). Befunde zu Assoziationen zum Weiterbildungsbegriff zeigen zudem, dass Lernen nicht zwangsläufig als lebenslange Angelegenheit betrachtet wird. Vielmehr steigt mit zunehmenden Alter die Auffassung, ,genug gelernt“ zu haben (ebd., S. 71).

Unsere These von der Lebensphasenabhängigkeit von Weiterbildung wird auch von Ergebnissen der Langzeitstudie von Harry Friebel et al. (2000) über Bildungsund Weiterbildungskarrieren einer Hamburger Schulabschlusskohorte 1997 gestützt. Das zentrale Ziel von Weiterbildung der untersuchten Kohorte ist deutlich stärker auf berufliche Identität (,,im Berufsleben bestehen“), weniger auf personale Identität (,geistig anregen“) ausgerichtet, d. h. die Lebensphase, in der man berufstätig ist, dominiert die Weiterbildungsentscheidungen dieser Kohorten. Auch in dieser Studie wird deutlich, dass die Bedeutung von Weiterbildung mit zunehmendem Alter abnimmt. Dies zeigt sich daran, dass dann jene Weiterbildungsaktivitäten im Vordergrund stehen, die in Arbeitgeberhand liegen, also nicht in eigener Veranlassung praktiziert werden (vgl. Friebel 2008, S. 111 ff.). Und schließlich hebt Maier-Gutheil (2015, S. 5) in ihrer Zusammenschau von internationalen und nationalen Studien zu Lernen und Bildung im Lebenslauf von Erwachsenen die Bedeutung von Lebenslaufkontexten des Lernens hervor und verweist damit indirekt auf die Lebensphasenabhängigkeit non-formalen und informellen Lernens. Mindestens zwei der dort genannten Lebenslaufkontexte (Familie, Ausbildung, Beruf, Weiterbildung, Freizeit) sind lebensphasenabhängig, drei, wenn man Familie als generative Eltern-Kind-Konstellation versteht.

Als Fazit lässt sich ein gewisser Widerspruch zwischen Lebensphasenkonzepten auf der einen und Konzepten Lebenslangen Lernens auf der anderen Seite herausarbeiten: Während phasenbezogene Konzepte Lernen tendenziell stärker im Kindes- und Jugendalter und in der Nacherwerbsphase positionieren, verorten programmatische Konzepte des Lebenslangen Lernens Lernprozesse über die gesamte Biografie. Vor diesem Hintergrund ist es interessant, sich empirisch einer Phase im Leben zu vergewissern, die programmatisch zwar mehr und mehr als Lern- und Weiterbildungsphase adressiert wird ${ }^{2}$, nach herkömmlichen (,normalbiografischen“, s. o.) Maßstäben aber nicht als Alter des Lernens verstanden wird: die Altersphase zwischen 50 und 60 Jahren, in der sich momentan die Angehörigen der sog. „Babyboomer-Generation“ befinden.

\subsection{Lebensphasen- und Altersbilder der Babyboomer}

Die Altersstruktur in Deutschland ist, bei allen u. a. durch Binnenwanderungen verursachten regionalen Unterschieden, von den „Babyboomern“, d. h. von den zwischen 1954 und 1964 geborenen Kohorten dominiert. ${ }^{3}$ Diese geburtenstarken Jahrgänge, die man durchaus als einen ,wesentlichen Akteur des momentanen gesellschaftlichen Alterungsprozesses in Deutschland“ (Dörner und Schäffer 2014a, S. 118) bezeichnen

\footnotetext{
${ }^{2}$ 50+ Programme der Bundesregierung: http://www.perspektive50plus.de/bundesprogramm. Vgl. hierzu: Knuth et al. 2014.

${ }^{3}$ In Deutschland wird es zukünftig mehr ältere Menschen (50+) geben, als jüngere. So sind Deutschland und Japan mit einem Medianalter von derzeit 45,7 bzw. 45,8 Jahren die Länder mit der ältesten Bevölkerung der Welt (vgl. Grünheid und Fiedler 2013)
} 
kann, befinden sich kollektiv in einer Lebensphase, die durch Innehalten, Rück- und auch Vorausblick gekennzeichnet ist. Charakteristisch für diese Lebensphase sind retrospektive Bilanzierungen des eigenen Lebens im Hinblick auf familiäre und berufliche Entwicklungen und daran anschließende Überlegungen, wie man den nächsten Lebensabschnitt gestaltet (ebd.). In Anlehnung an historische Lebenstreppendarstellungen (vgl. Ehmer 2007) kann man von einer „Plateauphase“ (vgl. Dörner und Schäffer 2014b) sprechen: Nach einem mit Anstrengungen verbundenen Lebensaufstieg in den Jahren der familiären und beruflichen Etablierung setzt in dieser Phase ein Moment des Verharrens und der Reflexion ein (vgl. ebd., S. 135).

Babyboomer werden allein aufgrund ihrer großen Zahl Einstellungen und Haltungen zu Lern- und (Weiter-)Bildungsprozessen in der zweiten Lebenshälfte, zum Übergang in die Nacherwerbsphase und zum Altern prägen. Unter Lebensphasengesichtspunkten sind vor allem die „Alters-, Alterns- und Altenbilder“ (Schäffer 2010; s. u.: 2.2) dieser Kohorten interessant, da mit ihnen auch Bilder und Vorstellungen über lebensphasenbezogene Angemessenheit von Weiterbildungsaktivitäten verbunden werden. Dies steht in Übereinstimmung mit der breiten Debatte über Altersbilder, die davon ausgeht, dass die unterschiedlichen Lebensalter sozial konstruiert sind und nicht nur in Bezug auf Weiterbildungsorientierungen viele Handlungsdispositionen beeinflussen (zum Altersbilddiskurs vgl. exemplarisch: Filipp und Mayer 1999; Göckenjan 2000; BMFSFJ 2010).

\subsection{Fragestellung}

In dem von der Volkswagenstiftung geförderten Forschungsprojekt „Weiterbildungsbeteiligung und Altersbilder der Babyboomer" (WAB) ${ }^{4}$ lag der Fokus auf der Frage nach Weiterbildungs-, Lern- und Bildungsorientierungen der Babyboomer in Abhängigkeit von deren Altersbildern und im Vergleich zu jüngeren und älteren Kohorten (vgl. Dörner und Schäffer 2014a; Krämer 2014). In dem vorliegenden Beitrag beschäftigen wir uns mit der Frage, inwiefern lebensphasenbezogene Orientierungen von Babyboomern mit Entscheidungen für oder gegen Weiterbildungsaktivitäten zusammenhängen.

\section{Sample, Methodik und Methodologie}

\subsection{Sample}

Im Mittelpunkt der Untersuchung, deren Haupterhebungsphase zwischen den Jahren 2010 und 2012 stattfand, standen die um 50-jährigen Kohorten. Um Vergleichshorizonte zu generieren, wurden Angehörige von Kohorten der um 30- und der um 70-Jährigen hinzugezogen. Es wurden insgesamt 46 Gruppendiskussionen mit 166 Personen durchgeführt. Die Diskussionszeit betrug zwischen 45 und 120 Minuten. Die Zielaltersgruppe bildeten die Babyboomer (*1954-1964; 76 Personen), die von jüngeren

\footnotetext{
${ }^{4}$ Das Projekt wurde von der VW-Stiftung unter dem Aktenzeichen 83191 zwischen den Jahren 2010 und 2013 gefördert.
} 
Tab. 1 Zusammensetzung des Samples nach Einzelpersonen, vgl. Dörner und Schäffer 2014a, S. 127

\begin{tabular}{|c|c|c|c|c|}
\hline & $\begin{array}{l}\text { Jüngere } \\
1965-1991 \\
21-47 \text { jährige } \\
n=73 \\
\end{array}$ & $\begin{array}{l}\text { „Babyboomer“ } \\
1954-1964 \\
48-58 \text { jährige } \\
n=76\end{array}$ & $\begin{array}{l}\text { Ältere } \\
\text { 1931-1953 } \\
\text { 59-81jährige } \\
n=17 \\
\end{array}$ & $\begin{array}{l}\text { Gesamt } \\
n=166\end{array}$ \\
\hline \multicolumn{5}{|l|}{$\overline{\text { Geschlecht }}$} \\
\hline männlich & 29 & 38 & 7 & 74 \\
\hline weiblich & 44 & 38 & 10 & 92 \\
\hline \multicolumn{5}{|l|}{ Bildungsabschluss } \\
\hline niedrig & 1 & 2 & 0 & 3 \\
\hline mittel & 21 & 42 & 6 & 69 \\
\hline hoch & 37 & 26 & 9 & 72 \\
\hline ohne Angabe & 14 & 6 & 2 & 22 \\
\hline \multicolumn{5}{|l|}{ berufliche Position } \\
\hline leitende Angestellte & 13 & 10 & 1 & 24 \\
\hline ausführende Angestellte & 47 & 27 & - & 74 \\
\hline Beamte & - & 7 & - & 7 \\
\hline Arbeiter & 5 & 12 & - & 17 \\
\hline Selbstständige & 5 & 11 & 2 & 18 \\
\hline erwerbslos & 1 & 6 & 9 & 16 \\
\hline Ruhestand & - & - & 5 & 5 \\
\hline ohne Angabe & 2 & 3 & - & 5 \\
\hline
\end{tabular}

Zusammensetzung des qualitativen Samples (Stichjahr 2012 nach Personen)

(*1965-1993; 73 Personen) und älteren Gruppen (*1931-1953; 17 Personen) flankiert wurden. Der Zugang zu den Gruppen erfolgte über unterschiedliche Kontaktpersonen (Schneeballprinzip). Es überwiegen Personen mit hohen und mittleren Bildungsabschlüssen (Tab. 1).

\subsection{Methodik und Methodologie}

Um handlungsleitende Orientierungen im Hinblick auf Alters-, Alterns- und Altenbilder zu erfassen, kamen Bildanalysen (vgl. Dörner 2012) und Gruppendiskussionen (vgl. Bohnsack 2007; Schäffer 2012a) zum Einsatz. Dabei verstehen wir Altersbilder als Vorstellungen über unterschiedliche Lebensalter, Alternsbilder als Vorstellungen über den Prozess des Alterns und Altenbilder als Vorstellungen über Menschen in höheren Lebensaltern (vgl. Schäffer 2010). Wir nehmen an, dass diese Vorstellungen über Alter, Altern und Alte in konkret gelebter und erfahrener Praxis (bspw. in Alltag und Beruf) angeeignet werden. Das so gewonnene Handlungswissen orientiert in impliziter Weise Alltags- und Berufshandeln sowie z. B. auch Bildungs-, Lernund Weiterbildungshandeln. Dies fußt auf einer methodologischen Annahme über die Unterscheidung von kommunikativem und konjunktivem Wissen: Kommunikatives Wissen umfasst institutionalisiertes und zweckrational organisiertes Wissen und kann daher von Akteuren kommunikativ expliziert werden. Konjunktives Wissen hingegen wird praxisgebunden von Akteuren angeeignet und inkorporiert und besitzt dann in Form von Habitualisierungen wiederum eine praxisorientierende Funktion (vgl. Bohnsack 2006a, S. 137). Wesentlich ist dabei, wie etwas gesagt oder getan wird, d. h., in welchem ,modus operandi der körperlichen und sprachlichen Praktiken“ (Bohnsack 
2006b, S. 132). Dazu wird rekonstruiert, wie Akteure bspw. Wirklichkeitskonstruktionen über Alter, Altern und Alte herstellen.

Empirisch haben wir Diskussionsgruppen unterschiedlichen Durchschnittsalters in einer mittleren Phase der Diskussion Fotos von Lehr-Lernsituationen mit Personen unterschiedlichen Alters vorgelegt, die diese besprechen sollen (,Photogruppendiskussionen“ vgl. Michel 2006; Schäffer 2010, S. 220 f.). Zudem wurden die Diskussionsteilnehmer angeregt, sich über ihre Alters, Alterns- und Altenbilder sowie über eigene Lern-, Bildungs- und Weiterbildungserfahrungen auszutauschen. Die Interpretation dieser Daten mittels der dokumentarischen Methode (vgl. Bohnsack 2007; Schäffer 2012b; Loos et al. 2013) erlaubt es, kollektiv geprägte „Denk- und Erfahrungsbilder" (Schäffer 2010) der Gruppen zu rekonstruieren. Diese wurden typisiert und flossen in eine rekonstruktive Theoriebildung zum Zusammenhang von Altersbildern mit Orientierungen im Hinblick auf Lebenslanges Lernen und mit Bildungs- und Weiterbildungsorientierungen ein.

\section{Babyboomer auf der Plateauphase: Weiterbildung als Selbstverständlichkeit, Schicksal, Marginalie oder Utopie?}

Wie schon dargelegt, befinden sich die 1954 und 1964 geborenen Babyboomerkohorten insgesamt in einer Lebensphase, die wir „Plateauphase“ genannt haben (vgl. 1.3), also einer Lebensphase, in der man auf sein bisheriges Leben bilanzierend zurückschaut und auch Pläne für die Zukunft macht. Je nach Lebenszusammenhängen gestaltet sich dieser Blick in unterschiedlicher Weise und hat auch Einfluss auf zukünftige Weiterbildungsentscheidungen dieser Kohorten. In unserem Sample konnten wir acht differierende Weiterbildungsorientierungen voneinander unterscheiden. Diese ließen sich in vier Obertypen einteilen, die wir nach Berufsstatus und formalem Bildungsgrad aufgeteilt haben (vgl. Abb. 1):

- Gruppen vom Obertypus Weiterbildung als Selbstverständlichkeit sind im Vergleich eher beruflich etabliert und von ihren Bildungsabschlüssen im mittleren bis höheren Bereich einzuordnen.

- Als maximaler Kontrast fungieren Gruppen des zweiten Obertypus Weiterbildung als Schicksal: Sie sind in ihrer Extremform arbeitslos und verfügen über niedrige Bildungsabschlüsse.

- Gruppen, die wir dem dritten Obertypus Weiterbildung als Marginalie zugeordnet haben, verfügen über einen formal eher niedrigen Bildungsgrad, sind aber beruflich vergleichsweise etabliert, d. h. befinden sich in sicheren Beschäftigungsverhältnissen.

- Schließlich sind Gruppen des vierten Obertypus Weiterbildung als Utopie solche mit vergleichsweise hohem Bildungsabschluss, aber unsicheren und teilweise prekären beruflichen Situationen.

Die vier Obertypen lassen sich als ein Orientierungsspektrum darstellen, bei dem „Weiterbildung (WB) als Selbstverständlichkeit“ und „WB als Schicksal“ zwei Extremausprägungen bilden. 


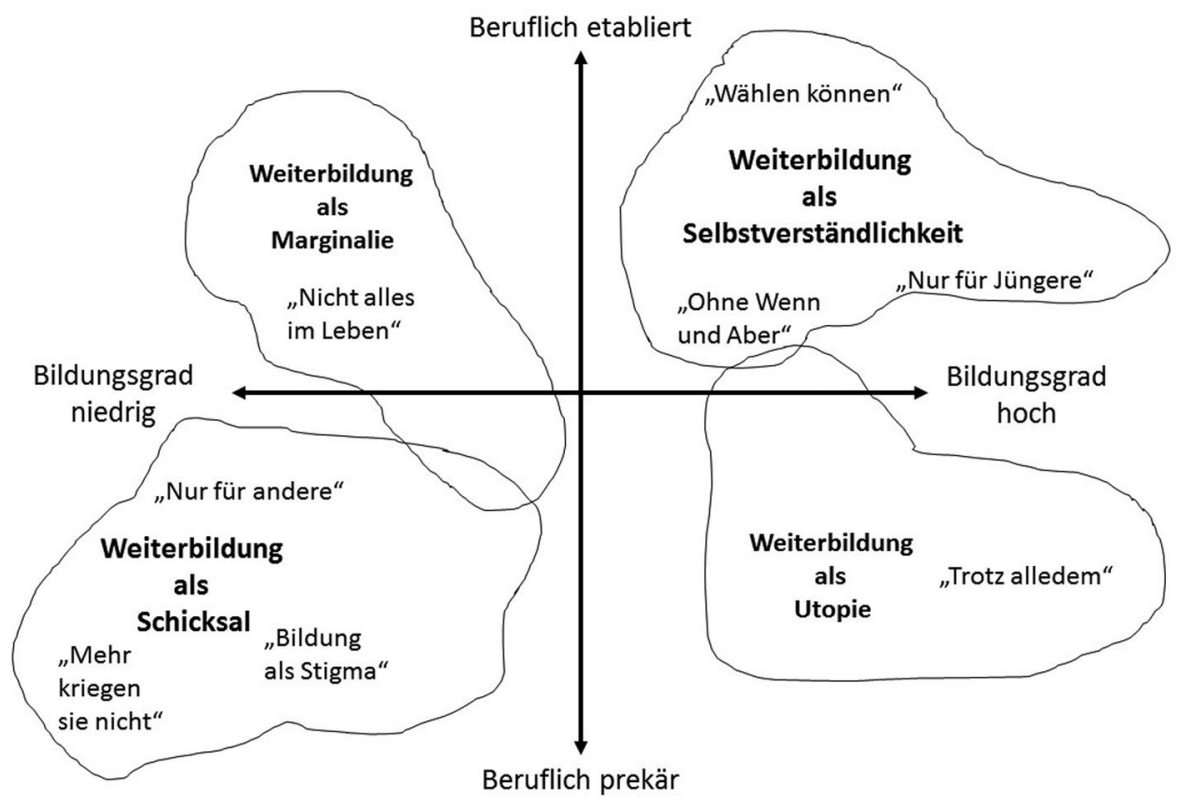

Abb. 1 Weiterbildung als Selbstverständlichkeit, Schicksal, Marginalie oder Utopie (einschließlich der acht Weiterbildungsorientierungen)

Anhand empirischer Fälle konnten wir bei allen Weiterbildungsorientierungen auch eine Lebensphasenabhängigkeit herausarbeiten. Je nach Typus wird diese allerdings unterschiedlich akzentuiert. Sie ergibt sich aus den milieuspezifischen Orientierungen zur Erfolgsträchtigkeit von Lernen in höheren Lebensaltern und der Wahrnehmung der eigenen Lebensposition: Wird diese eher als „sicher“ oder als „unsicher" aufgefasst? Zudem werden die Wahrnehmungen vom eigenen und fremden Lebensalter innerhalb erfahrungsgebundener Altersbilder gedeutet, in die auch Vorstellungen über ,typische“ Lebens- und Lernphasen eingelagert sind. Im Folgenden charakterisieren wir die acht Weiterbildungsorientierungen vor dem Hintergrund ihrer Einbindung in das skizzierte Viererspektrum.

\subsection{Weiterbildung als Selbstverständlichkeit}

Die drei Selbstverständlichkeitsorientierungen „Wählen-Können“, „Ohne Wenn und Aber“, und „Nur für Jüngere“ zeichnen sich durch eine generell hohe Weiterbildungsaffinität und eine grundlegende Akzeptanz von beruflicher und allgemeiner WB aus. Zentral ist die Wahrnehmung, dass man sich in der gegenwärtigen beruflichen Lebensphase für oder gegen WB relativ autonom entscheiden kann. Die Untertypen unterscheiden sich bei der Ausgestaltung dieses Autonomiespektrums: Bei der Ausprägung „Wählen können“ dominiert die autonome Entscheidungsfindung und bei der Ausprägung „Ohne Wenn und Aber“ ist die Entscheidungsfreiheit im Hinblick auf berufliche Anforderungsprofile eingegrenzt. Schließlich delegiert die Ausprägung „Nur für Jüngere“ Weiterbildung in einem autonomen Entscheidungsakt an Jüngere. 


\subsection{1 „Wählen-Können“}

Gruppen des Untertypus „Wählen-Können“ repräsentieren die extremste Ausprägung des Typus „WB als Selbstverständlichkeit“. Sie sind in akademischen Berufen etabliert und verfügen über Hochschulabschlüsse. Sie zeichnet aus, dass Weiterbildung nicht primär in einen beruflich-ökonomischen Begründungszusammenhang gestellt wird. Gleichwohl spielt der Beruf als relevanter Lebenskontext insofern eine Rolle, als dass er zumindest mit beruflicher Weiterbildung symbiotisch verschmolzen ist. Weiterbildung wird, entweder weil sie gesetzlich verpflichtend ist, oder aufgrund bestimmter Professionsselbstverständnisse, als periphere berufliche Tätigkeit angesehen. Dementsprechend geht es bei dem Untertypus um das „Auswählen“ von Inhalten und Zeitpunkten, die situativ und biografisch passen müssen. Weiterbildung ist damit auch lebensphasisch organisiert, insofern Beruflichkeit als zentrale Phase dreigeteilter Lebenslaufregimes gilt (vgl. 1.1).

Der Untertypus konnte u. a. in Kombination mit einem Altersbild eines gelassenen Blicks auf das kommende Alter empirisch rekonstruiert werden. Dieses beinhaltet, alterungsbedingte Einschränkungen zwar zu antizipieren, sie jedoch als üblich zu normalisieren und sich an dem in dieser Lebensphase Möglichen zu orientieren. Die Basis dafür bildet ein übergeordneter Orientierungsrahmen, der sich zwischen den Polen „Entfaltung“ und „Behinderung der Persönlichkeit“ aufspannt. Behinderung steht dabei für die gesundheitliche und kognitive Unbill des Alter(n)s. Entfaltung hingegen - als auf Individualismus und Distinktion verweisender Horizont - bedeutet „Weiterkommen“ im Leben und berufliche Entwicklung ,in der ganzen Breite“. Geeignetes Mittel für die berufliche Entwicklung ist Weiterbildung, die „voranbringen“ und gleichsam helfen soll, „den Bedürfnissen der Kundschaft gerecht zu werden".

Zusammen mit der Orientierungsdimension des Lernens wird das oben explizierte Altersbild stimmig. Alternsgelassen kann man sein, wenn man Alterserfahrenheit als Lern- und Bildungsvorteil deutet, der insbesondere bei der Wahl der subjektiv richtigen Entfaltungs-Möglichkeit weiterhilft. So wird Lernen zwar als ,altersbedingt differierende Fähigkeit“ (Krämer 2014, S. 153) interpretiert. Deren physiologisch verwurzelte Verschlechterung mit zunehmendem Alter wird jedoch durch Alterserfahrenheit nicht nur ausgeglichen, sondern sogar verbessert. Dem quantitativen Lernen der Jüngeren steht so das qualitative Lernen der Älteren gegenüber (vgl. zu dieser „umdeutend-akzeptierenden Lernorientierung“: Krämer 2014).

\subsection{2 „Ohne Wenn und Aber“}

Von Gruppen dieses Typus, die ebenfalls über höhere Bildungsabschlüsse verfügen, wird berufliche Weiterbildung als eine Pflicht „ohne Wenn und Aber“ gerahmt. Im Bereich allgemeiner Weiterbildung hingegen wird stärker selektiert. Die Selektion ist weniger auf ein individuelles Weiterkommen wie bei „Wählen-Können“ ausgerichtet, als vielmehr darauf, „was ei'm Spaß macht“. Dabei wird deutlich, dass die Lebenssphären Beruf und Privatleben in einem übergeordneten Rahmen getrennt voneinander behandelt werden und nicht wie bei „Wählen-Können“ im Brennpunkt des Ideals persönlicher Entfaltung verschmelzen. Grenzen der Selbstverständlich- 
keit von Weiterbildung werden im Blick auf das jeweilige Verhältnis von Aufwand zu Nutzen verhandelt. Damit einher geht allerdings ein Primat des Interesses, dem auch eine lernpositive Orientierung entspricht, d. h. eine betont affirmative Orientierung am Lernen, die zwischen biologischem Lernvermögen und motivationaler Lernbereitschaft differenziert und dabei Letztere als Garant des Lernerfolgs fokussiert. Insbesondere das Alter spielt dabei keine Rolle. Dies korrespondiert mit einem Altersbild, in dem die jetzige Lebensphase als Plateauphase eine Zeit zum „, Genießen " darstellt.

\subsection{3 „Nur für Jüngere“}

Vertreter dieses Untertyps verfügen über mittlere bis hohe Bildungsabschlüsse, weiterführende Berufsqualifikationen (z. B. Techniker, Meister) und sind in leitenden Positionen beruflich tätig. Weiterbildung gilt ihnen als selbstverständliches Moment der beruflichen Etablierung im jungen Erwachsenenalter und damit einhergehend der existenziellen wie familiären Verwirklichung. Die Notwendigkeit von Weiterbildung wird für diese Lebensphase nicht bezweifelt. Allerdings gilt Weiterbildung auch als Zumutung, der sich nicht verweigert werden darf, will man Beruf und Familie als Lebensaufgaben meistern. Man müsse nicht nur wollen, sondern es komme darauf an, „wie viel man bereit is, persönlich dafür zu opfern“. Zentraler und deutlich auf eine Dreiteilung des Lebenslaufs im Sinne Kohlis (vgl. 1.1) bezogener Rahmen solcher Gruppen ist, sich in früheren bzw. jungen Jahren ,genug“ weitergebildet zu haben. Die Vereinbarkeit von Weiterbildung mit den eigenen Lebensentwürfen schwindet, sobald die (Lebens-)Pflicht erfüllt ist und für die Kür Alternativen bestehen, die notfalls für argumentative Zwecke als Bildungsaktivitäten gerahmt werden können.

Die Selbstverständlichkeit, mit der Weiterbildung für sich selbst gewählt und für Jüngere gefordert werden kann, resultiert aus der Gewissheit eigener Lebenserfahrung gegenüber Jüngeren sowie einem Altersbild, wonach Altern nach den Pflicht- und Genussphasen des Lebens mit zunehmender körperlicher und geistiger Beeinträchtigung verbunden ist. So gestalte sich Lernen im Alter zwar schwieriger, jedoch habe man im Verlauf des Lebens Wissen erworben und Erfahrungen gemacht, die als Vorteil gegenüber Jüngeren gesehen werden.

\subsection{Weiterbildung als Schicksal}

Bei den drei Schicksalsorientierungen „Mehr kriegen sie nicht“, „Bildung als Stigma“ und „Nur für Andere“ steht die berufliche Existenzfrage im Mittelpunkt: Hier ist es vor allem berufliche WB, die nicht in der eigenen Hand liegt, sondern als abhängig von äußeren, nicht beeinflussbaren Umständen gerahmt wird. Die Ausprägungen des Typus unterscheiden sich dementsprechend in der Ausgestaltung des Spektrums von Heteronomieerfahrungen: „Mehr kriegen sie nicht“ und „Nur für andere“ stehen für erfahrungsgesättigte, mehr oder weniger resignative Haltungen Weiterbildung gegenüber. Es bestehen keinerlei Illusionen über die Möglichkeiten, noch weitere (berufliche) Weiterbildung zu bekommen. Gruppen der Ausprägung „Bildung als Stigma“ dagegen verwehren sich grundsätzlich gegen die Vorstellung, dass Weiter- 
bildung überhaupt sinnvoll ist, sehen sie aber als fremdbestimmte Notwendigkeit, um ,in Arbeit zu kommen“.

\subsection{1 „Mehr kriegen sie nicht“}

Dieser Untertypus rekrutiert sich aus Personen, die erwerbslos sind und deren Hoffnung sich darauf richtet, mittels Weiterbildung der (Langzeit-)Arbeitslosigkeit zu entkommen. Dementsprechend ist oft eine recht hohe Weiterbildungsbeteiligung anzutreffen: zuerst krankheitsbedingte Umschulungsmaßen, später dann Lehrgänge mit anschließenden obligatorischen Praktika o. Ä. Typischerweise münden diese Weiterbildungsmaßnahmen jedoch nicht in einen Arbeitsplatz. Dessen ungeachtet wird Weiterbildung nicht per se abgelehnt. Es wird die Auffassung vertreten, zu Beginn der Erwerbslosenkarriere in den späten 1990er Jahren die falsche Umschulung absolviert zu haben und die mangelnde Unterstützung durch das Arbeitsamt bei der Suche nach einer anderen Umschulungsmaßnahme beklagt: „Sie haben eine Umschulung gehabt. Mehr kriegen Sie nicht". Eine altersbezogene Selbstverortung wird vom Fehlen der tagesstrukturierenden Sicherheit eines Arbeitsplatzes und damit verbundenen Selbstverständlichkeit einer lebenszyklischen Eingebundenheit dominiert. Erzählungen und Beschreibungen kreisen um den schlechten Gesundheitszustand, der bestimmte Arbeiten nicht mehr erlaubt und um das beinahe schon verzweifelte Bemühen um alternative Tagesstrukturierungen (,viermal die Woche Sport“). Überraschenderweise fühlen sich diese Gruppen jünger als ihr chronologisches Alter. Diese Art des kontrafaktischen Jüngerfühlens - man ist eigentlich krank und hat auch keine altersangemessene Beschäftigung - kommt als Altersphasenbild nur bei den Erwerbslosen vor. Im Umkehrschluss kommt hier die lebensphasenstrukturierende Bedeutung von Erwerbstätigkeit zum Tragen. Die Orientierungen lassen sich als eine Art „soziales Altersvakuum“ beschreiben, bei dem die lebensphasenzyklische Strukturierung durch Erwerbsarbeit fehlt. Lernen wird von diesen Gruppen positiv gerahmt: „man kann alt werden wie ne Kuh und lernt immer noch dazu“. Je älter man werde, desto eher habe man „Lust (...) was Neues zu lernen“. Es wird hier ein geradezu verzweifeltes Bemühen deutlich, wenigstens über „Lernen“ an Gesellschaft teilzuhaben, wenn es denn nicht durch „Arbeit“ geht. Auch „Bildung“ wird von diesen Gruppen stark als Medium der Partizipation (,mitreden können“) und der Selbstbehauptung (,man kann sich eher behaupten“) gerahmt.

\subsection{2 „Bildung als Stigma“}

Auch diese Gruppen sind in unsicheren bis prekären Beschäftigungsverhältnissen, haben niedrige oder keine Bildungsabschlüsse und sind z. T. arbeitslos. Weiterbildung wird als Manko gesehen, da man entweder nicht in Arbeit kommt, weil man nicht die richtigen Abschlüsse hat oder umgekehrt Gefahr läuft, zu überfordernden Tätigkeiten herangezogen zu werden, wenn man eine Weiterbildung absolviert hat.

Der Gegensatz von „Arbeiten-Können vs. Bildungstitel“ ist bei Gruppen dieses Typus zentral. Je höher der Bildungsabschluss einer Person, desto weniger wird dieser Person zugetraut, arbeiten zu können. „Früher“ habe es ,auf dem Bau“ auch Arbeiter ohne Schulabschluss gegeben, die ,arbeiten konnten“. Hingegen seien die 
Abiturienten - „Überkandidelte“-, nicht zu gebrauchen gewesen. „Arbeiten können“ und „Bildung“ schließen sich in dieser Orientierungsfigur gegenseitig aus. Dieses Prinzip habe „früher“ (zu DDR-Zeiten) gegolten. Heute dagegen seien für eine Einstellung Bildungszeugnisse wichtig, die aber nichts über die Fähigkeiten der Inhaber aussagten. „Arbeiten-Können“ und „Arbeiten-Dürfen“ werden als zwei mitunter sich ausschließende Dinge gehandhabt. Die Idealvorstellung von „Arbeiten“ zeigt sich in der Verbindung von „Können“ und „Bildung“: „Du musst wat könn’n (...) bißchen wat in die Birne ham, bißchen vernünftich erzähln könn'n“. Bildung äußert sich hier als geistige Fähigkeit für alltägliches Miteinander, als Basis für Interaktion und Kommunikation.

Das Altersphasenbild von Gruppen diesen Typs bestimmen Orientierungen an einem ,angenehmen Arbeiten“, d. h. an Arbeit, die nicht „kaputt macht“ und ein „Leben nach der Arbeit“ im Sinne eines „Feierabends“ zulässt. Auch hier zeigt sich eine skeptische bzw. ablehnende Bildungsorientierung. Zuviel Bildung kann auch zu viel Arbeit bedeuten, denn: „wer viel kann, muss viel machen.“ „Lernen“ erweist sich klar als altersphasenabhängiges Konstrukt: Lernen im Alter wird, auch aufgrund negativer Erfahrungen mit Lernen im Jugendalter, ausgeschlossen bzw. auf Sprachenlernen begrenzt. Bildung ist in dieser Orientierung in zweifacher Hinsicht stigmatisiert: Entweder verhindere Bildung Arbeiten („Überkandidelt““ vs. „ohne Schulabschluss“) oder gutes Leben (,Wer viel kann, muss viel machen“).

\subsection{3 „,Nur für andere“}

Gruppen diesen Untertyps sind beruflich in unteren betrieblichen Positionen verankert, fühlen sich dort relativ sicher und insgesamt in ihrer Lebensphase zufrieden. Die Gefahr einer möglichen Arbeitslosigkeit wird gesehen, ist jedoch von untergeordneter Bedeutung. Die Grundzufriedenheit, die sich aus der Einschätzung speist, beruflich wie privat angekommen zu sein (,gut jetzt hab ich das Level jetzt grad erreicht und da bleibe ich auch dabei“) bietet keinen Anlass für Neuanfänge. Im Gegenteil werden solche nicht mehr für notwendig erachtet: „weil irgendwie mit 50, brauchst Du glaube ich, nicht mehr irgendwo was anderes machen". Aufgrund der Zufriedenheit mit dem bisher Erreichten, das auf einer subtilen, an chronologischen Lebensaltern und an normalbiografischen Entwürfen festhaltenden Lebensphasenorientierung aufruht, und der Risikowahrnehmung ihrer derzeitigen Erwerbssituation zeigt sich in Bezug auf Altern eine Durchhalteorientierung: Es geht darum, den Stand zu halten bzw. bis zum Ruhestand durchzuhalten. Allerdings - ganz im Sinne o. g. Schicksalshaftigkeit - wird Offenheit bekundet gegenüber sich ergebenden Gelegenheiten, die man ,am Schopfe packen“ würde. Weiterbildung, die im Zusammenhang mit beruflicher Verbesserung gesehen wird, könnte eine solche Gelegenheit sein, ergibt sich aber nicht. Vielmehr fühlen sich die Betreffenden weitestgehend von ihr ausgeschlossen und empfinden es als ungerecht, dass ,immer nur Andere“ im Unternehmen sich weiterbilden können. Weiterbildung als Möglichkeit beruflicher Verbesserung oder Karriere ist positiv belegt, bleibt aber unerreichbar. 


\subsection{Weiterbildung als Marginalie oder Utopie}

Zwischen diesen beiden Extremen sind die Typen „WB als Marginalie“ und „WB als Utopie“ angesiedelt. Für den Typus „WB als Marginalie“ stellt sich die Existenzfrage nicht in der Dringlichkeit, wie bei den Schicksalsgruppen, da hier relativ sichere Erwerbssituationen vorliegen. „Nicht alles im Leben“ kennzeichnet dementsprechend eine Haltung, bei der WB als eine zwar bekannte, aber für das eigene Handeln nicht relevante, eben marginale Handlungsoption gesehen wird. Dies stellt sich beim letzten Typus, „WB als Utopie“, anders dar. Hier wird WB nach wie vor akzeptiert und als zukünftige Möglichkeit gerahmt, obwohl negative Erfahrungen mit WB vorliegen. Insofern wird das prinzipielle Potenzial von WB, gewissermaßen kontrafaktisch (,Trotz alledem“) zu bisherigen negativen Erfahrungen, als hoch eingeschätzt - durchaus im Sinne einer positiven Utopie. Dies ist der zentrale Unterschied der Utopie- zu den Schicksalsgruppen, die keinerlei positiven Utopie im Hinblick auf WB anhängen.

\subsection{1 „Nicht alles im Leben“}

Gruppen des Untertypus „Nicht alles im Leben“ verfügen eher über mittlere Bildungsabschlüsse und befinden sich in etablierten beruflichen Positionen, die den Bildungsabschlüssen entsprechen. Weiterbildung wird weder als Selbstverständlichkeit, noch als Schicksal wahrgenommen, sondern als Marginalie. Das Thema ist für Gruppen dieses Typus ,abjeschlossen. (...) abjeschrieben. Vorbei.“ Die Orientierung ist allerdings weniger Ausdruck allgemeiner Resignation, als vielmehr einer lebensphasisch gerahmten, milieuspezifischen Marginalisierung von Weiterbildung. Konkret kann es sich dabei um berufliche und familiale Rahmungen, aber auch untergründige lebensphasenbezogene Lernorientierungen handeln: So befindet man sich in einer Phase relativer beruflicher Sicherheit, der zwar Veränderungs- und Aufstiegsmöglichkeiten fehlen. Das Erreichte möchte man jedoch durch vermeintlich vermessene Forderungen nach Weiterbildung und Weiterentwicklung nicht gefährden: „Aber kann ick mir abschminken. Ick kann froh sein, wenn ich da bleiben darf.“ Lebenssituationen mit jüngeren Kindern werden ebenfalls als Weiterbildungshemmnis interpretiert, denn „mich würd auch keiner nehm'n, denk ich mal, mit klein'n Kind“. Zudem befindet man sich bereits in einem Alter, in dem man ohnehin ,nisch mehr flexibel jenuch“ für die „Schulbank“ wäre.

Zwar verweist ein fallweise im Zusammenhang mit diesem Typus rekonstruiertes Altersbild der Ausblendung des chronologischen Alters oberflächlich auf die SelbstVerortung in einem Alters-Kontinuum. Doch Weiterbildung, Lernen und Bildung (als Erwerb institutioneller Abschlüsse) scheinen dem Kontinuum exterior zu sein. Dies kann auch erklären, warum die Weiterbildungsorientierung dennoch einen Altersphasenbezug aufweist. Er kann gesehen werden als milieuspezifisch gefärbte Plausibilisierung der Nicht-Teilnahme an dem, was zwar als Handlungsoption bekannt ist, jetzt aber außerhalb des eigenen Lebensphasenentwurfs liegt. Weiterbildung stellt in diesem Zusammenhang eine Marginalie dar, die von anderen Relevanzen überdeckt wird. 


\subsection{2 „,Trotz alledem“}

Vertreter dieses Untertyps sind gut ausgebildet (mittlere bis höhere Bildungsabschlüsse), befinden sich aber in beruflich unsicheren bis prekären Situationen - vornehmlich als Selbstständige in Berufen, die nicht ihrer Erstausbildung entsprechen. Sie sind weiterbildungserfahren und bezweifeln Weiterbildung nicht grundsätzlich (,Aktion $50+\ldots$ ist doch cool... die find die klasse“, ,gehört Weiterbildung zum täglichen Brot dazu"). Mehr noch: Es besteht ein fester Glaube an gute Weiterbildung, d. h. an solche, die professionell gut gemacht wird und neue berufliche Möglichkeiten bzw. Perspektiven eröffnen kann. Dieser Glaube steht im Zusammenhang mit mindestens einer guten Erfahrung und wird selbst dann nicht durch negative Weiterbildungserfahrungen erschüttert, wenn diese überwiegen. So werden Umschulungen als Chance für Neuanfänge gesehen, nicht aber als dauerhafte Prävention im Umgang mit Krisen und Risiken, die sich im Zuge von Neuanfängen (bspw. Selbstständigkeiten) immer wieder einstellen und sich mit zunehmenden Alter verschärfen. Trotz guter Gefühlslage hinsichtlich der eigenen Altersphase (,die heutigen 50-Jährigen sind die früheren 30-Jährigen") hadern die Betreffenden mit ihrer beruflichen Situation, sind von Existenzängsten getrieben und/oder unzufrieden. Im Gefühl, beruflich noch nicht erreicht zu haben, was in der Mitte des Lebens normalbiografisch eigentlich erreicht sein müsste, wird Weiterbildung zum festen Bestandteil im ständigen Bemühen um berufliche Konsolidierung (,kämpfen müssen“) oder Verwirklichung (,war's eigentlich nicht mein Traum, Musikschullehrer zu sein“). Kritisiert und beklagt wird Weiterbildung, die man besuchen müsse, um etwa einer Sperre von Sozialleistungen zu entgehen und mit der man nichts anfangen könne, da sie etwa jene Kompetenzen vermitteln sollte, die man in Form von Lebenserfahrungen bereits habe oder nicht zu einer Erwerbstätigkeit führen („Wie bewerbe ich mich richtig"). Dennoch oder trotz alledem wird Weiterbildung nicht verdammt, sondern bleibt bevorzugte Möglichkeit beruflicher Sicherung und Verbesserung und damit eine „Utopie“ für eine Zukunft, die immer näher kommt.

\subsection{Weiterbildung der Babyboomer im lebensphasenbezogenen Spannungsfeld} von selbstverständlicher Erfülltheit, schicksalhafter Resignation, utopischer Hoffnung und marginalisierter Anspruchslosigkeit

Insgesamt bestätigen unsere Befunde die Lebensphasenabhängigkeit von Weiterbildung, ihre Berufsbezogenheit und, vor allem in den von uns herausgearbeiteten Schicksalsorientierungen, ihren zumutenden resp. ausschließenden Charakter. Mehr noch zeigen sie aber, inwieweit berufliche und betriebliche Positionierungen im Zusammenspiel mit habitusspezifischen Wahrnehmungen der eigenen Lebenssituation Weiterbildungsorientierungen konstituieren. Hier spielen dann vor allem Einschätzungen und Interpretationen von Möglichkeiten und Chancen in Bezug auf Teilhabe an Bildung, Arbeit und Weiterbildung eine Rolle. So sind die Selbstverständlichkeitsorientierungen geprägt von zurückliegenden oder derzeitigen positiven Weiterbildungserfahrungen, die mit einem hohen Autonomieerleben im Beruf und z. T. mit einem verwirklichten Bildungsideal der Aufhebung der Trennung von beruflicher und privater Sphäre - und damit von beruflicher und allgemeiner Weiterbil- 
dung - einhergehen. Im Sinne der Institutionalisierung des Lebenslaufs (Kohli 1985) verkörpern diese Gruppen geradezu idealtypisch die schon in den Lebenstreppendarstellungen des 19. Jahrhunderts vorherrschende Vorstellung von einer lebensphasenspezifischen Erfüllung und Erfülltheit der Altersphase „um die 50“ (vgl. Ehmer 2007).

Gegenteilig sieht es bei den Schicksalsorientierungen aus, bei denen Weiterbildung überwiegend als heteronom erfahren wird. In eher prekären beruflichen Positionen oder in Arbeitslosigkeit verstrickt, kann Weiterbildung kaum souverän gehandhabt werden. Bildung allgemein wird tendenziell in ihrer Zugang zum Arbeitsmarkt ermöglichenden oder verhindernden Dimension gerahmt. Bezogen auf die Lebenslaufdimension lässt sich hier nicht von Erfüllung, wie beim zuerst genannten Typus sprechen, sondern von einem Kontinuum zwischen Akzeptanz, dass ,es so gekommen ist" (durchaus im Sinne des Bourdieuschen Notwendigkeitsgeschmacks) und einer lebensphasenbezogenen Resignation, die aufgrund ihrer zirkulären und selbstverstärkenden Struktur nur schwer von außen zu durchbrechen ist. Zwischen WB als Schicksal und WB als Selbstverständlichkeit sind die Orientierungen WB als Utopie und WB als Marginalie angesiedelt. Bei den Utopie-Orientierungen, die sich aus Gruppen mit hohem Bildungsstand und prekärer beruflicher Situation rekrutieren, überwiegt trotz schlechter WB-Erfahrungen der Glaube an die prinzipielle Sinnhaftigkeit von guter Weiterbildung. Lebensphasisch betrachtet, ist mit diesem Glauben die (z. T. kontrafaktische) Hoffnung verbunden, doch noch zu einer lebensphasenangemessenen Position zu gelangen (gewissermaßen dorthin, wo sich die Selbstverständlichkeitsgruppen bereits aufhalten). Schließlich spielt beim Typus WB als Marginalie, der sich aus beruflich etablierten, mit mittleren Bildungsabschlüssen versehenen Gruppen rekrutiert, WB überhaupt keine Rolle. Lebensphasenbezogen haben sich diese Gruppen von jeglichen beruflichen (Aufstiegs-)Ambitionen verabschiedet, sind damit aber keineswegs unzufrieden wie die Utopiegruppen oder resigniert, wie die Schicksalsgruppen. Vielmehr haben sie sich in ihrem Leben ,eingerichtet" und sind vor dem Vergleichshorizont der Selbstverständlichkeitsorientierungen als ,anspruchslos“ zu bezeichnen. Man könnte in Abwandlung des Mottos einer der Schicksalsgruppen (,Mehr kriegen sie nicht“) die Marginaliegruppen mit einem genügsamen „mehr wollen sie nicht“" charakterisieren.

\section{Schluss: Lernen in Lebensphasen - ein nach wie vor relevanter Zugang zum institutionalisierten Lernen Erwachsener}

Ausgegangen sind wir von einem Unbehagen an der These der ubiquitären Geltung Lebenslangen Lernens resp. lebenslanger Weiterbildung. Dieser These haben wir mit unseren empirischen Befunden ein lebensphasenorientiertes Modell gegenübergestellt, demzufolge Lernen von Individuen nach wie vor als eingebunden in unterschiedlich ausbuchstabierte, normalbiografische Vorstellungen gerahmt wird. Dies hindert Erwachsene zwar nicht, auch im späteren Lebensalter eine Weiterbildung zu beginnen. Allerdings geschieht dies tendenziell unter heteronomen Bedingungen von Beruf und Betrieb und damit auch nicht, wie oft dargestellt, über, ,intrinsische“ Lernmotivation oder weil es ganz ,normal“ geworden wäre, lebenslang zu lernen. Die- 
sem programmatischen Fehlschluss - weil alle über Lebenslanges Lernen räsonieren, wird Lebenslanges Lernen eine empirische Realität - haben wir versucht, mit unseren Ergebnissen und einer Re-Lektüre einschlägiger Studien etwas entgegenzusetzen. Zwar ist der Diskurs zum Lebenslangen Lernen allgegenwärtig, nicht aber Strukturen und Praxen lebenslangen Lernens und lebenslanger Weiterbildung. Wie wir aufzeigen konnten, schlagen sich die Diskurse nur bedingt in den Weiterbildungsorientierungen von Subjekten nieder und werden nur unter ganz spezifischen Bedingungen handlungswirksam. Auf diese Bedingtheit verweisen auch andere empirische Studien (vgl. Baethge und Baethge-Kinsky 2004; Schiersmann 2006; Reich-Claassen 2010; Friebel 2011). Allerdings ziehen diese Studien nicht die (radikale) Konsequenz, die Idee und in Teilen auch die Ideologie Lebenslangen Lernens einmal grundsätzlich zu hinterfragen.

Hier schließt sich eine weiterführende, den Rahmen dieses Beitrags übersteigende Frage an: Was bedeutet es für die Erwachsenenbildungswissenschaft, wenn sie ein Stück weit von dem überwiegend bildungspolitisch induzierten Paradigma des Lebenslangen Lernens abrückt und Weiterbildung (wieder) ausdrücklicher in ihrer Lebensphasenabhängigkeit reflektiert? Wir verstehen diesen Beitrag insofern als einen ersten Versuch, dem u. E. programmatisch überdimensionierten Projekt des Lebenslangen Lernens eine empirisch fundierte - und in diesem Sinne realistischere - Position des lebensphasenbezogenen Lernens gegenüberzustellen. Die Konsequenzen eines solchen Modells lebensphasenbezogenen Lernens für praktische Kontexte wären vermutlich geringer als die für den Diskurs des Lebenslangen Lernens selbst, da sich u. E. die Praxis keineswegs von einer Lebensphasenbezogenheit des formalen und non-formalen Lernens in Weiterbildungskontexten verabschiedet hat.

Open Access Dieser Artikel wird unter der Creative Commons Namensnennung 4.0 International Lizenz (http://creativecommons.org/licenses/by/4.0/deed.de) veröffentlicht, welche die uneingeschränkte Nutzung, Verbreitung und Wiedergabe für beliebige Zwecke erlaubt, sofern Sie den/die ursprünglichen Autor(en) und die Quelle ordnungsgemäß nennen, einen Link zur Creative Commons Lizenz beifügen und angeben, ob Änderungen vorgenommen wurden.

\section{Literatur}

Baethge, M., \& Baethge-Kinsky, V. (2004). Der ungleiche Kampf um das lebenslange Lernen. Eine Repräsentativ-Studie zum Lernbewusstsein und-verhalten der deutschen Bevölkerung. Münster: Waxmann.

Beck, U. (1986). Risikogesellschaft. Auf dem Weg in eine andere Moderne. Frankfurt a. M.: Suhrkamp.

Beck, U., \& Beck-Gernsheim, E. (1993). Nicht Autonomie, sondern Bastelbiographie. Anmerkungen zur Individualisierungsdiskussion am Beispiel des Aufsatzes von Günter Burkart. Zeitschrift für Soziologie ZfS, 22(3), 178-187.

Bilger, F., \& Kuper, H. (2013). Trendvergleich: Teilnahme und Aktivitäten. In F. Bilger, D. Gnahs, J. Hartmann, \& H. Kuper (Hrsg.), Weiterbildungsverhalten in Deutschland. Resultate des Adult Education Survey 2012 (S. 26-35). Bielefeld: W. Bertelsmann.

BMFSFJ - Bundesministerium für Familie, Senioren, Frauen und Jugend. (Hrsg.). (2010). Altersbilder in der Gesellschaft. Sechster Bericht zur Lage der älteren Generation in der Bundesrepublik Deutschland. Berlin: BMFSFJ.

Bohnsack, R. (2006a). Praxeologische Wissenssoziologie. In R. Bohnsack, W. Marotzki, \& M. Meuser (Hrsg.), Hauptbegriffe qualitativer Sozialforschung (S. 137-138). Opladen: Barbara Budrich.

Bohnsack, R. (2006b). Orientierungsmuster. In: R. Bohnsack, W. Marotzki, \& M. Meuser (Hrsg.), Hauptbegriffe qualitativer Sozialforschung (S. 132-133). Opladen: Barbara Budrich. 
Bohnsack, R. (2007). Rekonstruktive Sozialforschung. Einführung in qualitative Methoden. Opladen: Barbara Budrich.

Brandtstädter, J. (1990). Entwicklung im Lebenslauf. Ansätze und Probleme der Lebensspannen-Entwicklungspsychologie. In K.-U. Mayer (Hrsg.), Lebensläufe und sozialer Wandel (Kölner Zeitschrift für Soziologie und Sozialpsychologie KZfSS: Sonderheft 31, S. 322-350).

Brandtstädter, J. (2001). Entwicklung, Intentionaliät, Handeln. Stuttgart: Kohlhammer.

Dohmen, G. (1996). Das lebenslange Lernen. Leitlinien einer modernen Bildungspolitik. Bonn: Bundesministerium für Bildung, Wissenschaft, Forschung und Technologie.

Dörner, O. (2012). Bildanalysen in der Erwachsenenbildungsforschung. In B. Schäffer \& O. Dörner (Hrsg.), Handbuch Qualitative Erwachsenen- und Weiterbildungsforschung (S. 291-306). OpladenBerlin-Toronto: Barbara Budrich.

Dörner, O., \& Schäffer, B. (2014a). Sozialisation im Babyboom. Zeitschrift für Soziologie der Erziehung und Sozialisation ZSE, 34(2), 115-130. (In dies., Sozialisation im Babyboom).

Dörner, O., \& Schäffer, B. (2014b). Babyboomer auf der Plateauphase - ihre Altersbilder und Weiterbildungsorientierungen. Zeitschrift für Soziologie der Erziehung und Sozialisation ZSE, 34(2), 131146. (In dies., Sozialisation im Babyboom).

Ehmer, J. (2007). Die Lebenstreppe. In F. Jaeger (Hrsg.), im Auftrag des Kulturwissenschaftlichen Instituts (Essen) und in Verbindung mit den Fachherausgebern, Enzyklopädie der Neuzeit (Bd. 7, S. 882885), Stuttgart: J. B. Metzler.

Erikson, E. H. (1991). Wachstum und Krisen der gesunden Persönlichkeit. In E. H. Erikson (Hrsg.), Identität und Lebenszyklus (S. 55-122). Frankfurt a. M.: Suhrkamp.

Filipp, S.-H., \& Mayer, A.-K. (1999). Bilder des Alters: Altersstereotype und die Beziehungen zwischen den Generationen. Stuttgart: Kohlhammer .

Friebe, J., Schmidt-Hertha, B., \& Tippelt, R. (Hrsg.). (2014). Kompetenzen im höheren Lebensalter Ergebnisse der Studie ,C Competencies in Later Life“ (CiLL). Bielefeld: W. Bertelsmann.

Friebel, H. (2008). Die Kinder der Bildungsexpansion und das ,lebenslange Lernen “. Augsburg: Ziel.

Friebel, H. (2011) Weiterbildungsbeteiligung und Weiterbildungsmotivation. Hessische Blätter für Volksbildung, 3, 248-263.

Friebel, H., Epskamp, H., \& Knobloch, B. et al. (2000). Bildungsbeteiligung: Chancen und Risiken. Eine Längsschnittstudie über Bildungs- und Weiterbildungskarrieren in der „Moderne“. Opladen: Leske und Budrich.

Göckenjan, G. (2000). Das Alter würdigen. Altersbilder und Bedeutungswandel des Alters. Frankfurt a. M.: Suhrkamp.

Grünheid, E., \& Fiedler, C. (2013). Bevölkerungsentwicklung 2013. Daten, Fakten, Trends zum demografischen Wandel. Wiesbaden: Bundesinstitut für Bevölkerungsforschung.

Havighurst, R. J. (1972). Developmental tasks and education. New York: McKay (zuerst 1948).

Hof, C. (2009). Lebenslanges Lernen: Eine Einführung. Stuttgart: Kohlhammer.

Kade, J., \& Seitter, W. (1996). Lebenslanges Lernen. Mögliche Bildungswelten. Erwachsenenbildung, Biographie und Alltag. Opladen: Leske und Budrich.

Knuth, M., Stegemann, T., \& Zink, L. (2014). Die Wirkungen des Bundesprogramms „Perspektive 50plus. Chancen für ältere Langzeitarbeitslose“. IAQ-Report, 1.

Kohli, M. (1985). Die Institutionalisierung des Lebenslaufs. Historische Befunde und theoretische Argumente. Kölner Zeitschrift für Soziologie und Sozialpsychologie KZfSS, 37, 1-29.

Krämer, F. (2014). Wie lernen Babyboomer? Lernvermögen und Lernbereitschaft im Licht von Generation und Lebensphase. In O. Dörner \& B. Schäffer (Hrsg.), Sozialisation im Babyboom (Zeitschrift für Soziologie der Erziehung und Sozialisation ZSE: Schwerpunktthema, 34 Jg. H. 2, S. 149-164).

Kraus, K. (2001). Lebenslanges Lernen - Karriere einer Leitidee. Bielefeld: W. Bertelsmann.

Kuhlenkamp, D. (2010). Lifelong Learning. Programmatik, Realität, Perspektiven. Münster: Waxmann.

Kuwan, H., \& Seidel, S. (2013). Informelles Lernen Erwachsener. In F. Bilger, D. Gnahs, J. Hartmann, \& H. Kuper (Hrsg.), Weiterbildungsverhalten in Deutschland. Resultate des Adult Education Survey 2012 (S. 264-288). Bielefeld: W. Bertelsmann.

Leipold, B. (2012). Lebenslanges Lernen und Bildung im Alter. Stuttgart: Kohlhammer.

Leven, I., Bilger, F., Strauß, A., \& Hartmann, J. (2013). Weiterbildungstrends in verschiedenen Bevölkerungsgruppen. In F. Bilger, D. Gnahs, J. Hartmann, \& H. Kuper (Hrsg.), Weiterbildungsverhalten in Deutschland. Resultate des Adult Education Survey 2012 (S. 60-94). Bielefeld: W. Bertelsmann.

Lindenberger, U. (2002). Erwachsenenalter und Alter. In R. Oerter \& L. Montada (Hrsg.), Entwicklungspsychologie. Ein Lehrbuch (5. Aufl., S. 350-392). Weinheim: Psychologie Verlags Union. 
Loos, P., Schäffer, B., Przyborski, A., \& Nohl, A.-M. (2013). Dokumentarische Methode. Grundlagen Entwicklungen - Anwendungen. Opladen: Barbara Budrich.

Maier-Gutheil, C. (2015). Lern- und Bildungsprozesse im Lebenslauf - Befunde empirischer Forschung und Perspektiven der Theorieentwicklung. Zeitschrift für Weiterbildungsforschung, 1. doi:10.1007/ s40955-015-0008-y

Mayer, K. U., \& Diewald, M. (2007). Die Institutionalisierung von Lebensverläufen. In J. Brandtstädter \& U. Lindenberger (Hrsg.), Entwicklungspsychologie der Lebensspanne. Ein Lehrbuch (S. 510-539). Stuttgart: Kohlhammer.

Michel, B. (2006). Bild und Habitus - Sinnbildungsprozesse bei der Rezeption von Fotografien. Wiesbaden: VS-Verlag.

Nohl, A.-M., Rosenberg, F. v., \& Thomsen, S. (2015). Bildung und Lernen im biografischen Kontext. Empirische Typisierungen und praxeologische Reflexionen. Wiesbaden: Springer VS.

Pöggeler, F. (1964). Der Mensch in Mündigkeit und Reife. Eine Anthropologie des Erwachsenen. Paderborn: Ferdinand Schöningh.

Rausch, S. (2015). Lernen regierbar machen. Eine diskursanalytische Perspektive auf Beiträge der Europäischen Union zum Lebenslangen Lernen. Wiesbaden: VS-Verlag.

Reich-Claassen, J. (2010). Warum Erwachsene (nicht) an Weiterbildungsveranstaltungen partizipieren. Einstellungen und prägende Bildungserfahrungen als Regulative des Weiterbildungsverhaltens. Berlin: Lit Verlag.

Schäffer, B. (2010). Abbild - Denkbild - Erfahrungsbild. Methodisch-methodologische Anmerkungen zur Analyse von Alters-, Alterns und Altenbildern. In J. Ecarius \& B. Schäffer (Hrsg.), Typenbildung und Theoriegenerierung. Methoden und Methodologien qualitativer Bildungs- und Biographieforschung (S. 207-232). Opladen: Barbara Budrich.

Schäffer, B. (2012a). Gruppendiskussionsverfahren und Focus Groups. Geschichte, aktueller Stand und Perspektiven. In B. Schäffer \& O. Dörner (Hrsg.), Handbuch qualitative Erwachsenenbildungs- und Weiterbildungsforschung (S. 347-362). Opladen: Barbara Budrich.

Schäffer, B. (2012b). Dokumentarische Methode. Einordnung, Prinzipien und Arbeitsschritte einer praxeologischen Methodologie. In B. Schäffer \& O. Dörner (Hrsg.), Handbuch Qualitative Erwachsenen- und Weiterbildungsforschung (S. 196-2119). Opladen: Barbara Budrich.

Schlüter, A. (2010). Lernen in Lebensphasen. Karrierebiografien von Leiterinnen in Bildungseinrichtungen - oder: „Ich bin in einer Männerwelt sozialisiert worden“. Report. Zeitschrift für Weiterbildung, $4 / 2010,67-78$.

Schiersmann, C. (2006). Profile lebenslangen Lernens. Weiterbildungserfahrungen und Lernbereitschaft der Erwerbsbevölkerung. Bielefeld: W. Bertelsmann. 